\title{
Synchronization of spatially extended chaotic systems in the presence of asymmetric coupling
}

\author{
J. Bragard, ${ }^{1, *}$ S. Boccaletti, ${ }^{2}$ C. Mendoza, ${ }^{1,2}$ H. G. E. Hentschel,${ }^{3}$ and H. Mancini ${ }^{1}$ \\ ${ }^{1}$ Departamento de Física y Matemática Aplicada, Universidad de Navarra, E31080 Pamplona, Spain \\ ${ }^{2}$ Istituto Nazionale di Ottica Applicata, Largo Enrico Fermi 6, I-50125 Florence, Italy \\ ${ }^{3}$ Physics Department, Emory University, Atlanta, Georgia 30322, USA
}

(Received 24 February 2004; revised manuscript received 21 June 2004; published 30 September 2004)

\begin{abstract}
In a recent paper [Phys. Rev. Lett. 91, 064103 (2003)] we described the effects of asymmetric coupling configurations on the synchronization of spatially extended systems. In this paper, we report the consequences induced by the presence of asymmetries in the coupling scheme on the synchronization process of a pair of one-dimensional fields obeying complex Ginzburg-Landau equations. While synchronization always occurs for large enough coupling strengths, asymmetries have the effect of enhancing synchronization and play a crucial role in setting the threshold for the appearance of the synchronized dynamics, as well as in selecting the statistical and dynamical properties of the synchronized motion. We analyze the process of synchronization in the presence of asymmetries when the dynamics is affected by the presence of phase singularities, and show that defects tend to anchor one system to the other. In addition, asymmetry controls the number of synchronized defects that are present in the dynamics. Possible consequences of such asymmetry induced effects in biological and natural systems are discussed.
\end{abstract}

DOI: 10.1103/PhysRevE.70.036219

PACS number(s): 05.45.Xt, 05.45.Gg, 05.45.Jn

\section{INTRODUCTION}

The synchronization of coupled chaotic systems has been a topic of intense study since 1990 [1]. In this context, different type of synchronization have been described: identical and generalized synchronization, phase synchronization, lag and intermittent lag synchronization, and anticipating synchronization. Furthermore, synchronization effects have been explored in natural phenomena and laboratory experiments [2-5]. Unified approaches to describing and measuring synchronization states have also been proposed [6,7].

Recently, interest has moved to the study of synchronization phenomena in space-extended systems, such as large populations of coupled chaotic units and neural networks [8], globally or locally coupled map lattices [9-11], coupled map networks [12] as well as other space-extended systems [13-19].

In most cases, studies of chaotic synchronization have mainly considered external forcings, and bidirectional symmetric or unidirectional master-slave coupling configurations. In many practical situations, however, one cannot expect to have purely unidirectional, nor perfectly symmetrical coupling configurations. As a result, recent interest has focused on detecting asymmetric coupling configurations [20], and quantifying asymmetries in the coupling scheme in relevant applications (such as the study of the human cardiorespiratory system) [21], and then to characterize the effects of asymmetric coupling on synchronization (for example, between pairs of one-dimensional space extended chaotic oscillators [22]). In particular, Ref. [22] has shown that asymmetry in the coupling of two one-dimensional fields obeying complex Ginzburg-Landau equations (CGLE) enhances

\footnotetext{
*Electronic address: jbragard@fisica.unav.es
}

complete synchronization, and plays an important role in controlling the properties of the final synchronized dynamics.

In this paper, we present a detailed characterization of asymmetric coupling on the synchronization of a pair of non identical CGLE, for both small and large parameter mismatches. We will analyze the type of synchronized dynamics occurring in the presence of asymmetric coupling in all possible dynamical states emerging from CGLE, and we will show (i) that in all cases the threshold for the appearance of synchronized motion depends non trivially on the asymmetry in the coupling; (ii) the selection of the dynamics within the final synchronized manifold is always crucially affected by the asymmetry; (iii) the process leading to synchronization is anticipated by defect-defect synchronization, inducing the simultaneous appearance in the coupled fields of phase singularities, even in the cases in which the uncoupled dynamics of both fields does not include the presence of defects.

\section{THE MODEL}

We will consider a pair of one-dimensional fields obeying complex Ginzburg-Landau equations. This equation has been extensively investigated in the context of space-time chaos, since it describes the universal dynamical features of an extended system close to a Hopf bifurcation [23], and therefore it can be considered as a good model equation in many different physical situations, such as occur in laser physics [24], fluid dynamics [25], chemical turbulence [26], bluff body wakes [27], or arrays of Josephson's junctions [28]. A recent review on the CGLE [29] comprehensively describes the wide class of physical situations where CGLE represents a good model equation.

We will consider a pair of complex fields $A_{1,2}(x, t)$ $=\rho_{1,2}(x, t) e^{i \phi_{1,2}(x, t)}$ of amplitudes $\rho_{1,2}(x, t)$ and phases $\phi_{1,2}(x, t)$, whose dynamics obeys 


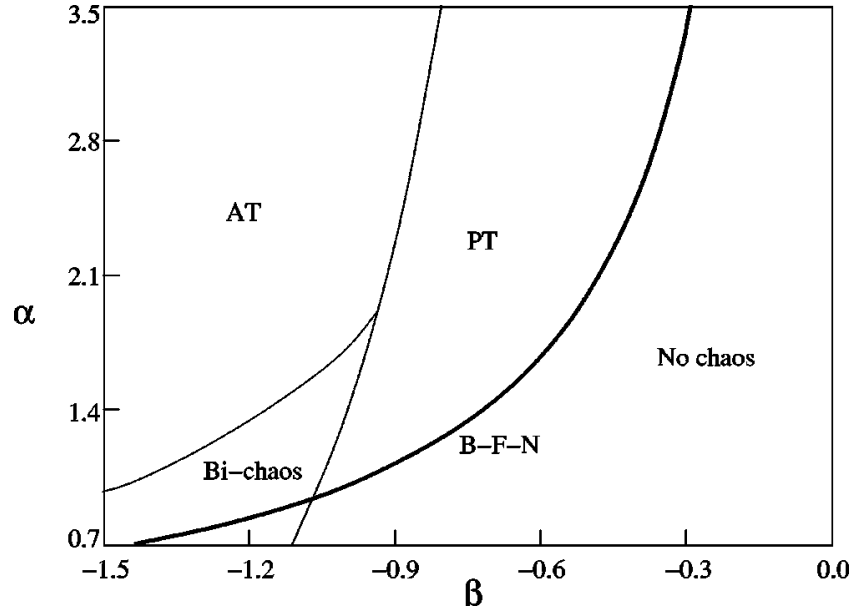

FIG. 1. $(\alpha, \beta)$ parameter space for Eq. (1) for $c=0$. The lines delimit the borders for each one of the dynamical regimes produced by Eq. (1), and the Benjamin-Feir-Newel line for stability of the plane wave solutions.

$$
\begin{aligned}
\dot{A}_{1,2}= & A_{1,2}+(1+i \alpha) \partial_{x}^{2} A_{1,2}-\left(1+i \beta_{1,2}\right)\left|A_{1,2}\right|^{2} A_{1,2}+\frac{c}{2}(1 \mp \theta) \\
& \times\left(A_{2,1}-A_{1,2}\right) .
\end{aligned}
$$

Here, overdots denote temporal derivatives, $\partial_{x}^{2}$ stays for the second derivative with respect to the space variable $0 \leqslant x \leqslant L$ ( $L$ being the system extension), $\alpha$ and $\beta_{1,2}$ are suitable real parameters, $c$ represents the coupling strength, and $\theta$ is a parameter accounting for the asymmetry in the coupling. The case $\theta=0$ describes the bidirectional symmetric coupling configuration, whereas the case $\theta=1(\theta=-1)$ recovers the unidirectional master-slave scheme, with the field $A_{1}$ $\left(A_{2}\right)$ driving the response of $A_{2}\left(A_{1}\right)$.

When $c=0$ (the uncoupled case), different dynamical regimes occur in Eq. (1) for different choices of the parameters $\alpha, \beta$ [30-32]. The full parameter space for the dynamics of the CGLE is shown in Fig. 1. In particular, Eq. (1) admits plane wave solutions (PWS) of the form

$$
A_{q}(x, t)=\sqrt{1-q^{2}} e^{i(q x+\omega t)}, \quad-1 \leqslant q \leqslant 1 .
$$

Here, $q$ is the wave number in Fourier space, and the temporal frequency is given by $\omega=-\beta-(\alpha-\beta) q^{2}$. The stability of such PWS can be analytically studied below the Benjamin-Feir-Newel (BFN) line (defined by $\alpha \beta=-1$ in the parameter space). Namely, for $\alpha \beta>-1$, one can define a critical wave number,

$$
q_{c}=\sqrt{\frac{1+\alpha \beta}{2\left(1+\beta^{2}\right)+1+\alpha \beta}},
$$

such that all PWS are linearly stable in the range $-q_{c} \leqslant q$ $\leqslant q_{c}$. Outside this range, PWS become unstable through the Eckhaus instability [33].

When crossing from below the BFN line in the parameter space, Eq. (3) shows that $q_{c}$ vanishes and all PWS become unstable. Above this line, Refs. [30-32] identify different turbulent regimes, called, respectively, amplitude turbulence (AT) or defect turbulence, phase turbulence (PT), bi-chaos, and a spatiotemporal intermittent regime. The borders in parameter space for each one of these dynamical regimes are schematically drawn in Fig. 1, together with the BFN line. In this work, we will mainly concentrate on PT and AT, since they constitute the fundamental dynamical states for the evolution of the uncoupled fields, and their main properties [34] have received considerable attention in recent years including the definition of suitable order parameters marking the transition between them [35], as well as the study of nonlinear structures (e.g., Nozaki-Bekki holes, modulated amplitude waves and homoclons) forming the "building blocks" of the nonlinear dynamics of the CGLE [36,37]. Related studies of synchronization phenomena in CGLE with bidirectional symmetrical coupling configurations can be found in Refs. [17,38-40].

PT is a regime where the chaotic behavior of the field is mainly dominated by the dynamics of $\phi(x, t)$, the amplitude $\rho(x, t)$ changing only smoothly, and being always bounded away from zero. On the other hand, AT is the dynamical regime wherein the fluctuations of $\rho(x, t)$ become dominant over the phase dynamics. Here, the complex field experiences large amplitude oscillations which can (locally and occasionally) cause $\rho(x, t)$ to vanish. As a consequence, at all those points (hereinafter called space-time defects or phase singularities) the global phase of the field $\Phi$ $\equiv \arctan [\operatorname{Im}(A) / \operatorname{Re}(A)]$ shows a singularity. Phase singularities have been proved to play a crucial role in the description of the dynamics of optical [41], fluid [42], and chemical [43] experiments as well in cosmology [44]. Furthermore, the 2003 Nobel prize for physics has been awarded for researches in the field of superconductors and superfluids, that can be described phenomenologically with the help of the CGLE [45].

\section{METHODS AND INDICATORS}

The purpose of our paper is to report the different synchronization states that are selected when asymmetrically coupling exists between the two CGLE fields. In order to be as exhaustive as possible, we will consider different regimes for the two CGLE. The reference as a starting point is the case treated in Ref. [22] (i.e., $\alpha=2, \beta_{1}=-0.7$, and $\beta_{2}=$ $-1.05)$. For this parameter choice, the two fields are originally prepared to display PT and AT, respectively. As a consequence, hereinafter we will denote this situation as PTAT(I). Another possible choice for an initial PT-AT configuration, whose relevance will be momentarily clear, is to consider $\alpha=2, \beta_{1}=-0.95$, and $\beta_{2}=-1.2$ [we will denote such a situation as PT-AT(II)]. Finally, we will consider also cases of small parameter mismatch, where the two systems start from the same initial dynamical state, such as $\alpha=2$, $\beta_{1}=-0.75$, and $\beta_{2}=-0.9$ (denoted by PT-PT) and $\alpha=2, \beta_{1}$ $=-1.05$, and $\beta_{2}=-1.2$ (denoted by AT-AT).

In all cases, we consider values of the asymmetry parameter $\theta \in[-1,+1]$, and highlight the effects of asymmetry in the synchronization properties $(c \neq 0)$ of system 1. Simulations were performed with a Crank-Nicholson, AdamsBashforth scheme (which is second order in space and time [46]), with a time step $\delta t=10^{-2}$ and a grid size $\delta x=0.25$, for 


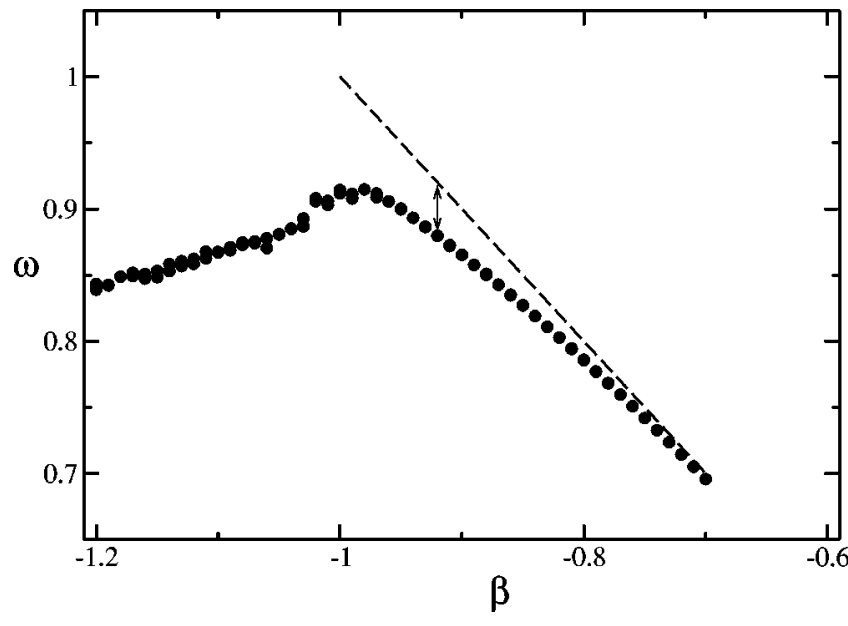

FIG. 2. Natural averaged frequency $\omega$ (see text for definition) vs $\beta$ for $\alpha=2$. The filled dots report the values from simulations of Eq. (1) at $c=0$. The dashed line $\omega=-\beta$ is the prediction given by the dispersion relation of the plane wave solutions with zero wave number.

$L=100$ (corresponding to 400 grid points) and spatial periodic boundary conditions $\left[A_{1,2}(0, t)=A_{1,2}(L, t)\right]$.

A crucial parameter in all our investigations, which dictated the choice of the parameters in the different cases, is the natural average frequency of the single CGLE. Such a frequency is calculated from the numerical simulations of a single CGLE by averaging in space the unfolded phase $\phi$ defined in $\mathbb{R}$ rather than in $[0,2 \pi]$. We have

$$
\omega=\lim _{t \rightarrow \infty} \frac{\langle\phi(x, t)\rangle_{x}}{t}
$$

where $\langle\cdots\rangle_{x}$ represents a spatial average.

Figure 2 shows $\omega$ vs the parameter $\beta$ at $\alpha=2$. In order to construct Fig. 2, we have integrated the CGLE for a very long time $\left(t_{f}=15000\right)$ after eliminating transient behavior $(T=5000)$. Two different initial conditions for each value of $\beta$ were chosen in order to measure the sensitivity of $\omega$ with respect to selection of different initial conditions. It should be emphasized that all initial conditions were chosen to have a zero average phase gradient [35], because the frequency in the PT regime is highly sensitive to the average phase gradient as shown by [35].

From Fig. 2 one clearly realizes that $\omega$ reaches a maximum for $\beta \approx-0.98$, close to the transition from the PT to the AT regime. This transition has been extensively studied by several authors $[35,47,48]$, and it has been shown that it depends on the spatial extension on which Eq. (1) is integrated, as well as on the average phase gradient. In addition, it is interesting to notice (see Fig. 2) that on the right-hand side of the maximum (PT regime) the two different initial conditions lead to nearly the same value for the averaged frequency, while on the left-hand side of the maximum (AT regime) the two initial conditions lead in general to two different values for $\omega$. This fact could serve as an alternative indicator for the characterization of the PT-AT transition. Furthermore, the frequency difference between the prediction given by the dis- persion relation of the PWS (dashed line) and the numerical simulations can be evaluated quite accurately in the PT regime (right-hand side of the maximum) by using the modified Kuramoto-Sivashinsky equation [49,50].

Considerations based on Fig. 2 dictate the choice for the parameters $\beta$ 's in the rest of the presentation. Indeed, a question to be clarified is how crucial is the role of the natural frequency for the selection of the dynamics for the two coupled CGLE in the synchronized state. A previous study with bidirectional symmetrical coupling configuration $(\theta$ $=0$ ) between a PT and a AT regime [17] pointed out that the final synchronized dynamics occurs in a PT state. The above result was obtained for a parameter choice for which the frequency $\omega_{\mathrm{PT}}$ of the initial PT state was smaller than the one $\left(\omega_{\mathrm{AT}}\right)$ of the initial AT state. This was also the situation of the case PT-AT(I) treated in Ref. [22] (see Fig. 2). We will show that, in the absence of asymmetries, the dynamics in the final synchronized state is always selected to correspond to that state having an originally smaller value of $\omega$. This property has dictated the choice of parameters for the case PT-AT(II) considered in the present paper $\left(\beta_{1}=-0.95\right.$ and $\left.\beta_{2}=-1.2\right)$. In this case Fig. 2 shows that $\omega_{\mathrm{PT}}>\omega_{\mathrm{AT}}$, and we will see that the synchronized motion at $\theta=0$ develops onto a AT regime.

Let us now discuss how to characterize the synchronization properties of the coupled fields by means of suitable indicators [19]. As we are dealing with extended chaotic fields that may be in defect turbulence, concepts of phase synchronization may be hindered by the presence of phase singularities in such regimes, that makes average phases difficult to be properly defined.

On the other hand, complete synchronization (CS) states can be detected by the use of Pearson's coefficient defined as

$$
\gamma=\frac{\left\langle\left(\rho_{1}-\left\langle\rho_{1}\right\rangle\right)\left(\rho_{2}-\left\langle\rho_{2}\right\rangle\right)\right\rangle}{\sqrt{\left\langle\left(\rho_{1}-\left\langle\rho_{1}\right\rangle\right)^{2}\right\rangle} \sqrt{\left\langle\left(\rho_{2}-\left\langle\rho_{2}\right\rangle\right)^{2}\right\rangle}}
$$

where \langle\rangle denotes a full space-time average (in order to avoid getting spurious values, we allow in general some transient time $T$ to elapse before evaluating this coefficient). $\gamma$ measures the degree of cross correlation between the moduli $\rho_{1}(x, t)$ and $\rho_{2}(x, t)$ : When $\gamma=0$ the two fields are linearly uncorrelated; while $\gamma=1$ marks complete correlation and $\gamma$ $=-1$ indicates that the fields are negatively correlated.

Another indicator characterizing the disorder in the system is the number of phase singularities (or defects) $N$. Theoretically, a defect is a point $(x, t)$ for which $\rho(x, t)=0$. This implies that defects are intersections of the 0 -level curves in the $(x, t)$ plane of the real and imaginary parts of $A_{1,2}(x, t)$. In practice, because of the finite size of the mesh and of the finite resolution of the numerics, we must introduce a method for the detection of a defect. A reliable criterium is to count as defects at time $t$ those points $x_{i}$ where the $\rho\left(x_{i}, t\right)$ is smaller than 0.025 and that are furthermore local minima for the function $\rho(x, t)$.

It is well known $[47,48]$ that $N$ is an extensive quantity of both time and space, and therefore it is sometimes convenient to refer to the defect density $n_{D}$, that is calculated as the defect number $N$ per unit time and unit space. Anticipating 
the results of Sec. VI, it appears that the defects of system 1 and system 2 tend to anchor each other in the synchronized states.

In the following, we will describe the important effects of asymmetries in the coupling of system 1 , for different values of the parameters $\beta_{1}$ and $\beta_{2}$, while $\alpha=2$ will be hereinafter fixed.

\section{ASYMMETRY ENHANCED SYNCHRONIZATION}

A striking effect of asymmetry in the coupling that has already been highlighted in our previous analysis for the case PT-AT (I) [22] is that one can improve dramatically the synchronization threshold by selecting a suitable level of asymmetry in the coupling. Conversely, one can also achieve desynchronization of the two coupled systems by varying the asymmetry level in the coupling scheme.

\section{A. Large parameter mismatch}

By selecting in (1) a sufficiently large parameter mismatch in the equations for $A_{1,2}$, one can set the uncoupled evolutions of $A_{1}$ and $A_{2}$ to be in PT and AT, respectively. By doing that, one still has three possibilities of choosing the parameters $\beta$ accordingly to the natural frequencies of the two separate CGLE.

The first case [PT-AT(I)] corresponds to system 1 in the PT regime $\left(\beta_{1}=-0.7\right.$ with a lower natural frequency than system 2 in the AT regime $\left(\beta_{2}=-1.05\right)$. The natural frequencies are approximately equal to $\omega_{1} \approx 0.7$ and $\omega_{2} \approx 0.87>\omega_{1}$ (see Fig. 2). This situation has been extensively studied in Ref. [22] where both complete and frequency synchronization features were discussed and characterized.

The second case [PT-AT(II)] corresponds to preparing system 1 in the PT regime $\left(\beta_{1}=-0.95\right)$ with a higher natural frequency than system 2 in the AT regime $\left(\beta_{2}=-1.2\right)$. The natural frequencies are approximately equal to $\omega_{1} \approx 0.9$ and $\omega_{2} \approx 0.84<\omega_{1}$ (see Fig. 2). For this case, we will show how asymmetry enhances the setting of complete synchronization.

Notice that a further situation could be studied if the two systems are prepared in the PT and AT regimes, respectively, but they have approximatively the same natural frequency. This more complex case, where one might expect some kind of resonance coming into play in the process of synchronization, will be dealt with elsewhere.

Figure 3(a) reports $\gamma$ vs the parameter space $(c, \theta)$ for the PT-AT(II) case, and shows the nontrivial dependence of the threshold for synchronization on the asymmetry parameter $\theta$. A better way to visualize such a dependence is by making a cut of the surface at a fixed value of the coupling [e.g., $c$ $=0.25$, see Fig. 3(b)]. Both in the PT-AT(II) case and in the PT-AT(I) case (already reported in Fig. 1b of Ref. [22]), a better synchronization level is obtained for the unidirectional configuration where the system in the PT regime is driving the system in the AT regime $(\theta=1)$. The surfaces and curves of Fig. 3(a) and 3(b) have been obtained by making averages over a time $t_{f}=15000$ after a large transitory has elapsed
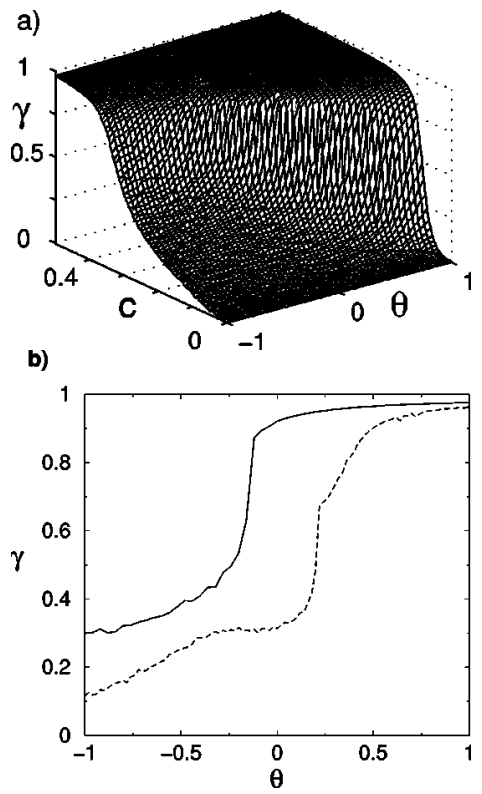

FIG. 3. (a) Pearson's coefficient $\gamma$ (see text for definition) vs the parameter space $(c, \theta)$. Other parameters are $\alpha=2, \beta_{1}=-0.95$, and $\beta_{2}=-1.2$ [case PT-AT(II)]. (b) Solid line, $\gamma$ vs $\theta$ [cut of the $\gamma$ surface in (a)] at $c=0.25$, highlighting the role of asymmetry in enhancing synchronization. The dashed line reports the same for the PT-AT(I) case already studied in Ref. [22].

$(T=6000)$ in order to ensure that we are measuring stationary synchronization states.

\section{B. Small parameter mismatch}

The very same scenario of asymmetry enhanced synchronization occurs when we select small parameter mismatches in Eq. (1), i.e., we set the parameters so as the two uncoupled fields are both either in PT or AT, thus confirming that this feature generally characterizes the emergence of the synchronized motion in our system.

\section{AT-AT case}

In this case, we set $\beta_{1}=-1.05$ and $\beta_{2}=-1.2$. Both systems now are in the AT regime, with system 1 having a natural frequency higher than the one of system 2.

Figure 4 shows Pearson's coefficient vs the parameter space $(c, \theta)(a)$, as well as a cut of the $\gamma$ surface at $c=0.17$ (b), showing that asymmetry in the coupling is still playing an important role in modifying the level of synchronization for a fixed value of the coupling strength $c$. It is not surprising that the complete synchronization threshold is now lower compared to the PT-AT cases. This, indeed, is related to the fact that smaller parameter mismatches induce closer initial dynamics, which are therefore easier to synchronize.

\section{PT-PT case}

Finally, in order to complete this first part of the discussion, we examine the PT-PT case. Now, parameters are $\beta_{1}$ $=-0.75$ and $\beta_{2}=-0.9$, determining an initial PT state for both 


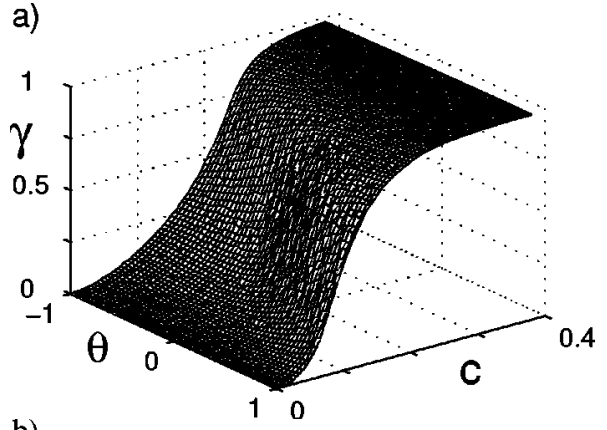

b)

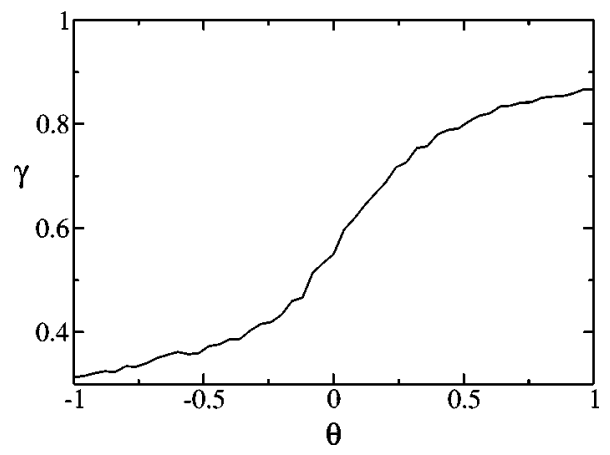

FIG. 4. (a) Pearson's coefficient $\gamma$ (see text for definition) vs the parameter space $(c, \theta)$. Parameters are $\alpha=2, \beta_{1}=-1.05$, and $\beta_{2}=$ -1.2 (AT-AT case). (b) $\gamma$ vs $\theta$ [cut of the $\gamma$ surface in (a)] at $c$ $=0.17$ showing the dependence of the synchronization threshold with asymmetry. The figure is obtained by using statistics over time $t_{f}=50000$ rather than $t_{f}=15000$ (as for the other figures), in order to have smoother curves.

uncoupled fields, with system 1 having a lower natural frequency with respect to system 2 .

Figures 5(a) and 5(b) describe the behavior of $\gamma$ as a function of the coupling $c$ and the asymmetry $\theta$. Once again, asymmetry plays a decisive role in enhancing the appearance of a synchronized motion in system 1 . Notice that here the values of $c$ required for a synchronized motion are smaller than in any of the previous cases, reflecting the fact that the present situation corresponds to the smallest parameter mismatch.

At variance with all the other cases, an interesting feature of Fig. 5(b) is that an increase in the asymmetry does not always yield a monotonic increase of $\gamma$. Anticipating the results of Sec. VI, this anomalous feature is due to a substantial number of defects being generated in both systems in the intermediate stage before synchronization, even if both dynamics were in the absence of coupling (at $c \equiv 0$ ) in the PT regime.

At this stage, we can already draw some interesting conclusions. We have seen that changing asymmetry in the coupling configuration for the same coupling strength has the effect of enhancing the appearance of a synchronized motion or destroying synchronization, regardless of the initial uncoupled state of the dynamics. We conjecture that this may have relevant consequences in biological systems, where changes in asymmetry of the interactions could be a way to efficiently synchronize-desynchronize the dynamics for the same strength of interaction. Some studies have indeed been

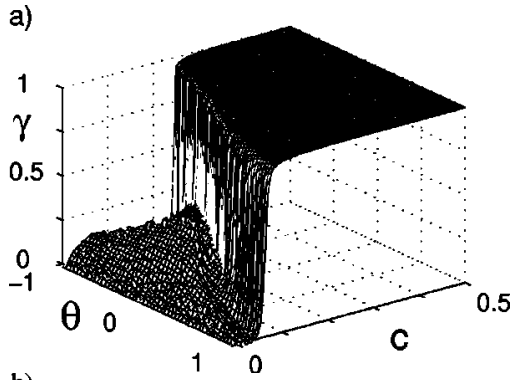

b)

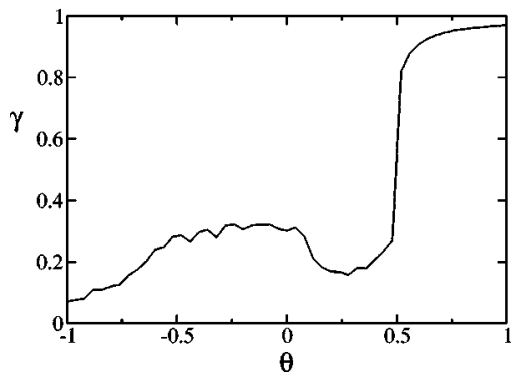

FIG. 5. (a) Pearson's coefficient $\gamma$ (see text for definition) vs the parameter space $(c, \theta)$. Parameters are $\alpha=2, \beta_{1}=-0.75$, and $\beta_{2}=$ -0.9 (PT-PT case). (b) $\gamma$ vs $\theta$ [cut of the $\gamma$ surface in (a)] at $c$ $=0.095$. In this case a total time $t_{f}=15000$ was used for the calculation of the spatiotemporal averages.

published $[5,51,52]$ indicating different modes of synchronization for animal populations.

Let us recall that in Eq. (1) the coupling is a mapping of all the grid points of system 1 on their corresponding grid points of system 2 . We could, in fact, imagine more complicated and probably more realistic configurations where couplings, besides being asymmetric, would be spatially dependent or even asynchronous. While it is likely that real systems show combinations of asymmetric, asynchronous and spatially dependent coupling schemes to control and synchronize in an optimal way their dynamical regimes, here we only focused on the effects of asymmetries, since the scenario of emerging dynamics is already extremely rich in this "simplified" approach.

\section{SELECTION OF THE FINAL STATE}

Next, we move to describe how asymmetries play a crucial role in setting the state of the dynamics within the synchronized regime, which occur for large values of the coupling strength. Let us recall the methods adopted for our investigation of the dynamics within the synchronized regime. Initially $(t=0)$ we begin a trial simulation of the two Eqs. (1) connected with a nonzero value of $c$. We impose random initial conditions on both systems, which in general will have different parameters. As a consequence, the dynamics usually attains synchronized motion only after a transient time $T$. Since we are not here interested in characterizing the dynamics in the transient stage, we let a certain transient time $T$ elapse (we have verified that $T=6000$ is large enough for reaching such asymptotic state) before starting to calculate the indicators of any asymptotic synchro- 

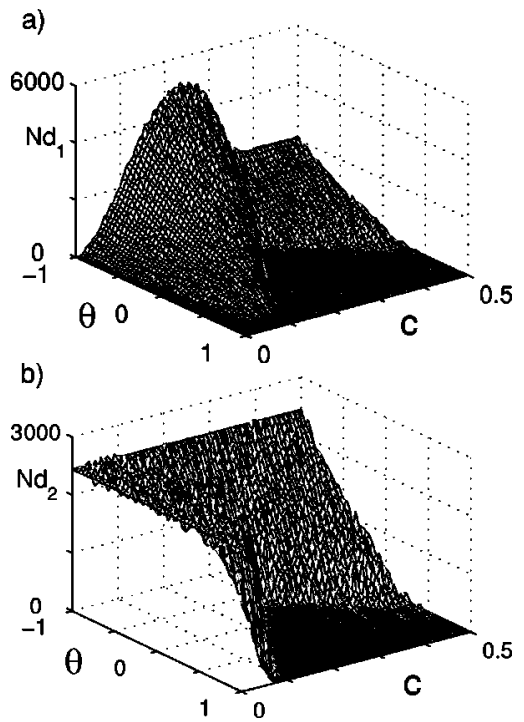

FIG. 6. Total number of defects counted during a time $t_{f}$ $=15000$ for the PT-AT(II) case. (a) Number of defects appearing in system 1, that was set initially in the PT regime at $c=0$; (b) Number of defects appearing in system 2, that was set initially in the AT regime at $c=0$.

nized state. In this way, we can measure such indicators within the statistically stationary state represented by the asymptotic synchronized motion.

While it is not surprising that when coupling two initially PT states (AT states) the final synchronized motion will persist in the PT regime (AT regime), a relevant point concerns what mechanisms control the selection of the synchronized motion, once the two fields originally start from different regimes. To address such an issue, we will focus in the present section on the two PT-AT cases. In these cases, it is not trivial to predict a priori what will be the resulting dynamical state for the synchronized motion.

Figures 6(a) and 6(b) show the total number of defects counted for a time $t_{f}=15000$ in the parameter space $(c, \theta)$ for the PT-AT(II) case. Namely, Fig. 6(a) [Fig. 6(b)] corresponds to the defects appearing in system 1 (in system 2) that was set initially in the $\mathrm{PT}$ regime (in the AT regime) at $c$ $=0$. One clearly sees that both systems exhibit a large number of defects for nonzero coupling. Furthermore, for asymptotically large values of the coupling $(c \approx 0.5)$ leading to a synchronized motion, the asymmetry parameter $\theta$ plays a crucial role in setting the synchronized dynamics on either a PT regime or an AT regime. The defect number vs. the parameter space for the case PT-AT(I) was already reported by us in Fig. 2 of Ref. [22], where again was emphasized the role of the asymmetry in the selection of the synchronized dynamical regime.

Let us compare and discuss more fully these two cases. In Sec. III, we have already seen that the main difference between the cases PT-AT(I) and PT-AT(II) is in terms of the initial natural frequencies of the two subsystems. Namely, in the PT-AT(I) [the PT-AT(II)] case the natural frequency of the subsystem originally set in AT is larger (smaller) than the

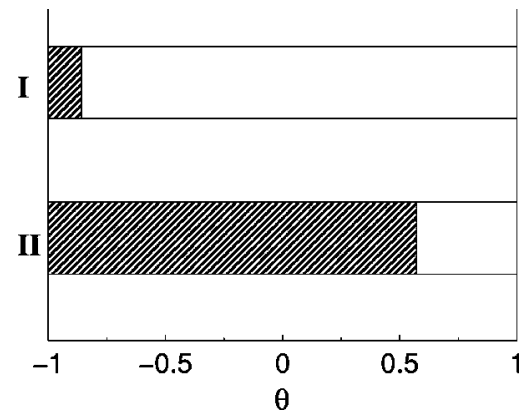

FIG. 7. Dynamical states of the synchronized motion (obtained for large values for the coupling strength $c$ ) attained by the two subsystems in the PT-AT cases. The upper (lower) bar corresponds to the PT-AT(I) [the PT-AT(II)]. The dashed zone refers to a common AT regime while the blank zone marks a common PT regime.

one of the subsystem originally set in PT. In Fig. 7 we summarize the result of the comparative study of the two cases. We choose a sufficiently large value of the coupling strength so as to ensure a synchronized state, and we have represented with a dashed region (a blank region) the range of $\theta$ values for which the synchronized motion develops into an AT (a PT) regime.

First of all we observe that at $\theta=0$ (i.e., in the bidirectional symmetrical case) the system with a lower natural frequency is the dominant one at the moment of selecting the final synchronized state. Furthermore, in Fig. 7 we observe a very different scenario for the two PT-AT cases. In the PTAT(I) case a final state in PT is selected for most of the values of the asymmetry parameter (until $\theta=-0.84$, below which a final state in AT takes over). In contrast, in the PTAT(II) case for most of the asymmetry values (up to $\theta$ $=0.64$ ) the final state is selected in the AT regime.

The conclusion of the present section is that asymmetries in the coupling configuration play a decisive role in the selection of the dynamics and the statistical properties of the synchronized state.

\section{DEFECT ANALYSIS}

In this last section we finally discuss the role of topological defects in the process of synchronization. Let us recall that a defect is defined as a point in the space-time plot where the modulus of the complex field is vanishing. In the present case, the spatial boundary conditions are periodic, which prevents defects from disappearing at the boundary (as is the case of rigid boundary condition). Our initial conditions are chosen so as to be free of topological defects. As a consequence, the constraint of charge conservation for the defects insures that the total charge stays zero at all subsequent times (up to the precision of the numerical calculations). In the following we analyze in detail the AT-AT and PT-PT cases. 

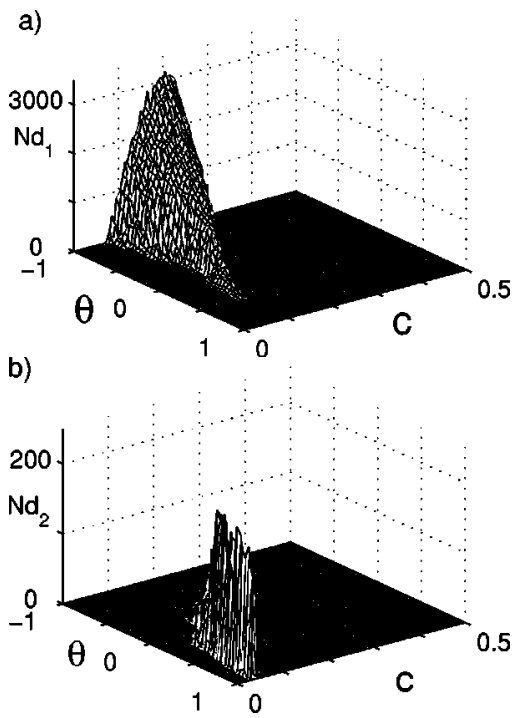
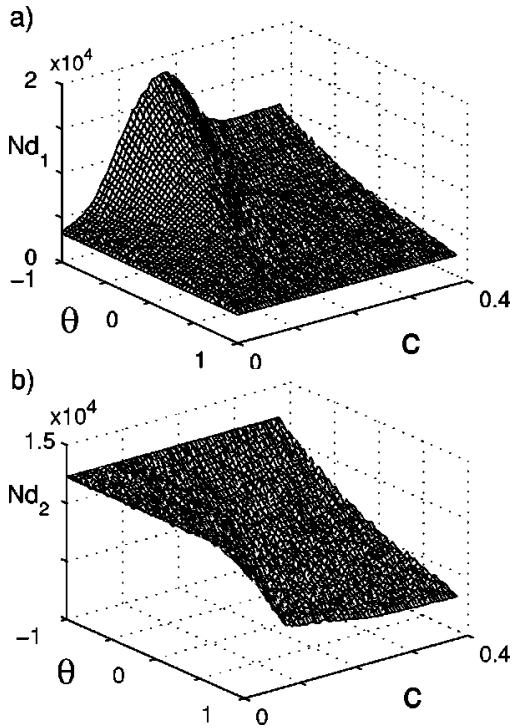

FIG. 8. Total number of defects counted during a time $t_{f}$ $=15000$ for the PT-PT case. (a) corresponds to system 1 with $\beta_{1}$ $=-0.75$; (b) corresponds to system 2 with $\beta_{2}=-0.9$.

\section{A. PT-PT case}

The PT-PT case corresponds to setting systems 1 and 2 in the PT regime with $\beta_{1}=-0.75$ and $\beta_{2}=-0.9$. Such a parameter choice implies that the natural frequency (at $c=0$ ) of system 1 is lower than the one of system 2 . This situation is particularly relevant because the two uncoupled systems have initially no defects, and the final synchronized state develops also in a PT regime without defects. Therefore one's intuition might lead one to conclude that the whole process of synchronization of the two subsystems would not be affected by the presence of phase singularities.

Figures 8(a) and 8(b) show instead that this is not the case. In fact a finite number of defects are produced for intermediate values of the coupling strength. Moreover, such a feature characterizes the synchronization process in the intermediate stages for all values of the asymmetry parameter. Obviously, when one of the systems does not receive inputs from the other (as occurs when it is the master in a masterslave configuration at $\theta= \pm 1$ ) then no defects are injected there all throughout the synchronization process. In contrast, when $\theta \neq \pm 1$, the appearance of defects characterize both subsystems during the whole process leading to synchronization.

Figures $8(a)$ and $8(b)$ show a further interesting feature of defect formation in the intermediate stage. Namely, we notice that the maximum number of defects in the subsystem having an initially lower natural frequency (system 1) is approximately an order of magnitude higher than the maximum number of defects in the subsystem having an initially higher natural frequency (system 2). Eventually, when the two subsystems give rise to a synchronized motion for large values of the coupling $(c>0.3)$, defects disappear, indicating that the synchronized dynamics takes place in a PT regime.

For $\theta= \pm 1$, we have a master-slave coupling scheme. In this condition, the master obviously imposes its dynamics on
FIG. 9. Total number of defects counted during a time $t_{f}$ $=50000$ for the AT-AT case. (a) corresponds to system 1 with $\beta_{1}$ $=-1.05$; (b) corresponds to system 2 with $\beta_{2}=-1.2$.

the slave subsystem, and it is to be expected that the average frequency of the synchronized dynamics equals the original frequency of the master system. However, when $\theta \neq \pm 1$, a nontrivial point to be discussed concerns the dependence of the average frequency of the synchronized state on the asymmetry in the coupling configuration. In order to explore this feature, we have set a sufficiently large coupling strength $(c=0.5)$ and we have checked that the frequency of the synchronized dynamics shows an approximately linear dependence in the asymmetry parameter $\theta$, starting from the frequency $\omega_{1}$ (corresponding to the original frequency of subsystem 1 at $c=0$ ) for $\theta=1$ and ending at the frequency $\omega_{2}$ (corresponding to the original frequency of subsystem 2 at $c=0)$ for $\theta=-1$. This indicates that for large couplings and intermediate values of the asymmetry, the synchronization process corresponds to an adjustment of the two subsystems onto a common rhythm of oscillation which is intermediate between the original different rhythms of the two spatial oscillators at $c=0$.

\section{B. AT-AT}

We finally consider the AT-AT case, where system 1 is originally in the AT regime at $\beta_{1}=-1.05$ and is characterized by a natural frequency higher than the one of system 2 (which is also originally in the AT regime at $\beta_{2}=-1.2$ ). In this situation, a substantial number of phase defects characterizes the initial states of both subsystems, the final synchronized dynamics, as well as all intermediate stages in the process of synchronization, which takes place by a mechanism of anchoring of phase singularities.

As shown in Figs. 9(a) and 9(b), the total number of defects in the two subsystems varies with the coupling and the asymmetry parameters. In particular, system 1 experiences a 


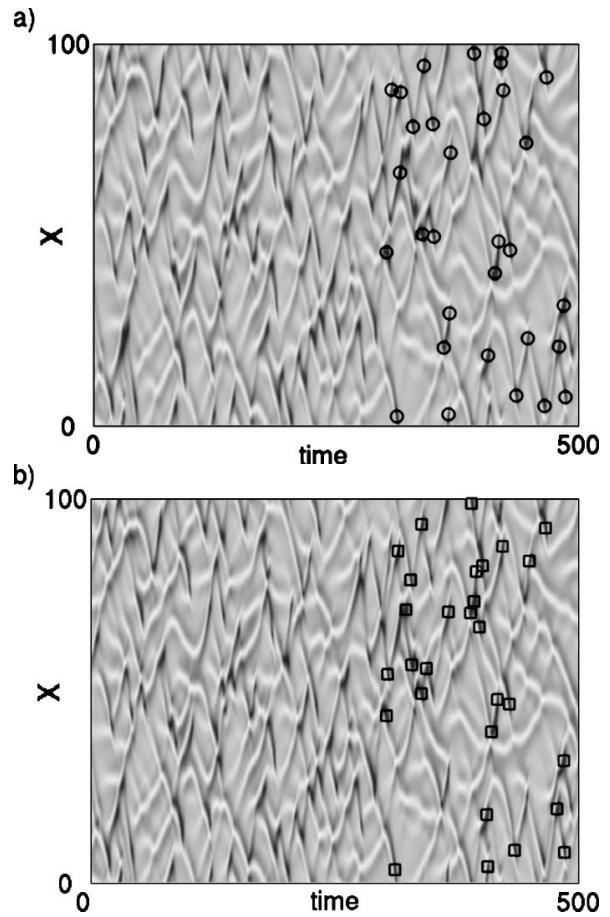

FIG. 10. Localization of the defects in the space (vertical) time (horizontal) representation of the modulus of $A$ in the AT-AT case $\left(\beta_{1}=-1.05\right.$ and $\left.\beta_{2}=-1.2\right)$. Other parameters are $c=0.3$ and $\theta=$ -0.88 . $\bigcirc$ indicates defects in system 1 (a) and $\square$ indicates defects in system 2 (b). Only defects for $t>300$ are displayed.

huge increase in its defect number (by nearly an order of magnitude) for intermediate values of the coupling $c \approx 0.18$, when it is slaved by system 2 . In contrast, the number of defects in the system 2 (whose initial natural frequency is lower) experiences a monotonic decrease when the coupling strength $c$ increases. For large values of $c$, the number of defects in the synchronized state depends almost linearly on the asymmetry starting from the original value of $N_{1}$ for system 1 in the absence of coupling for $\theta=1$ and ending to the original value of $N_{2}$ for system 2 at $\theta=-1$.

In order to visualize the anchoring mechanism of phase singularities during the process of synchronization, in Figs. 10 (a) and 10(b) we report the localization of the defects in both subsystems at an intermediate value of the coupling strength $(c=0.3)$ and for $\theta=-0.88$, showing that already at this intermediate stage of synchronization more than half the defects of system 1 are anchored with corresponding defects in system 2.

Finally, in Fig. 11 we present the total number of defects in system 1 (solid line), the total number of defects in system 2 (dashed line) and the total number of synchronized defects (dotted line) vs the coupling strength $c$ for $\theta=0.88$ [Fig. 11(a)] and $\theta=-0.88$ [Fig. 11(b)]. The number of synchronized defects (dotted lines in Fig. 11) is defined as the total number of defects having same localization $(x, t)$ in both subsystems.

From Fig. 11 one can infer that the synchronization process is accompanied by a gradual increase of the number of a)

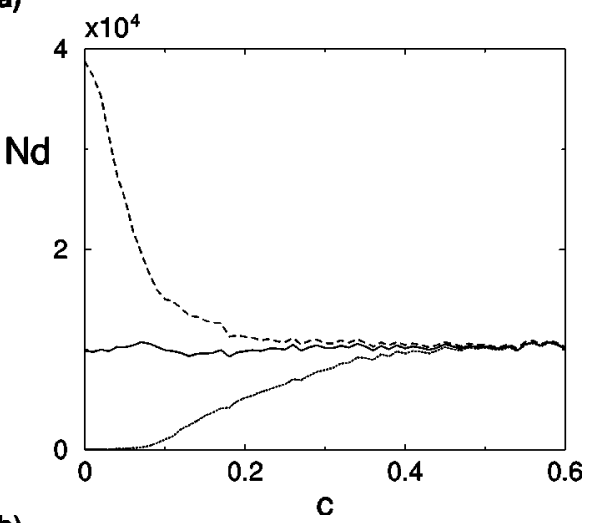

b)

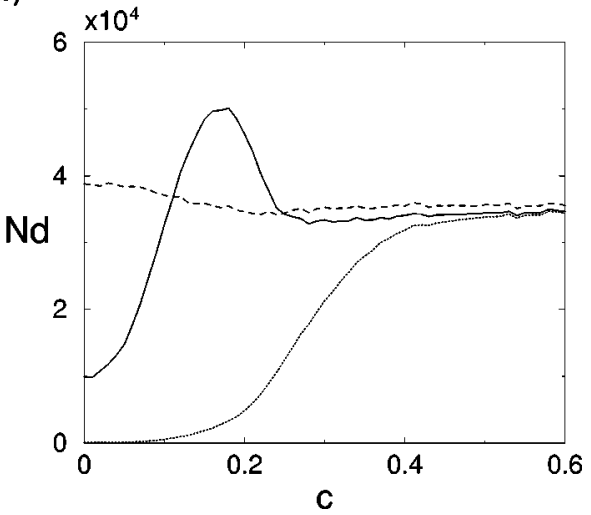

FIG. 11. Total number of defects in the system 1 (solid line), total number of defects in the system 2 (dashed line), and total number of synchronized common defects (dotted line) vs $c$ for the AT-AT case. (a) corresponds to $\theta=0.88$; (b) corresponds to $\theta=$ -0.88 .

synchronized defects, reflecting a progressive anchoring of phase singularities in the two subsystems, as the coupling strength increases.

\section{CONCLUSIONS}

In conclusion, we have reported and discussed several asymmetry induced effects in the process of synchronization of a pair of coupled complex space extended fields. While synchronization always occurs for large enough values of the coupling strength, the threshold for the setting of synchronized motion crucially depends on the asymmetry in the coupling configuration. Furthermore, the asymmetry controls in relevant cases the statistical and dynamical properties of the synchronized motion, as is the case when the coupled subsystems start from statistically different dynamical regimes. In this latter situation we have shown that a bidirectional symmetrical coupling configuration leads to a synchronized motion where the statistical properties of the subsystem having originally a lower natural frequency prevail, whereas asymmetries can drastically change such a scenario. Finally, we have studied the process of synchronization in the presence of asymmetries when all stages of the dynamics are affected by the presence of phase singularities, showing that 
defects tend to anchor from one system to the other, and that the asymmetry controls the number of synchronized defects that are present in the motion.

We argue that such features may have relevant consequences in biological and natural systems, where small changes in the asymmetry of the interactions could be used as an efficient way to synchronize or desynchronize the dynamics, as well as select the main statistical properties of the synchronized motion in ensembles of interacting complex units.

\section{ACKNOWLEDGMENTS}

This work was partly supported by EU Contract No. HPRN-CT-2000-00158 (COSYC of SENS), MIUR-FIRB Project No. RBNE01CW3M-001, and MCYT (Spain) Project No. BFM2002-02011 (INEFLUID).
[1] For a comprehensive review on the subject see S. Boccaletti, J. Kurths, G. Osipov, D. Valladares, and C. Zhou, Phys. Rep. 366, 1 (2002), and references therein.

[2] K. Kaneko and I. Tsuda, Complex Systems: Chaos and Beyond, A Constructive Approach with Applications in Life Sciences (Springer-Verlag, Berlin, 2000).

[3] A. Winfree, The Geometry of Biological Time (Springer, New York, 2001).

[4] S. H. Strogatz, Sync: The Emerging Science of Spontaneous Order (Hyperion Press, New York, 2003).

[5] S. Manrubia, A. Mikhailov, and D. Zanette, Emergence of Dynamical Order (World Scientific, Singapore, 2004).

[6] S. Boccaletti, D. L. Valladares, L. M. Pecora, H. P. Geffert, and T. Carroll, Phys. Rev. E 65, 035204 (2002).

[7] L. Pastur, S. Boccaletti, and P. L. Ramazza (unpublished).

[8] S. H. Strogatz, R. E. Mirollo, and P. C. Matthews, Phys. Rev. Lett. 68, 2730 (1992).

[9] V. N. Belykh and E. Mosekilde, Phys. Rev. E 54, 3196 (1996).

[10] M. Hasler, Yu. Maistrenko, and E. Mosekilde, Phys. Rev. E 58, 6843 (1998).

[11] A. Pikovsky, O. Popovych, and Yu. Maistrenko, Phys. Rev. Lett. 87, 044102 (2001).

[12] S. Jalan and R. E. Amritkar, Phys. Rev. Lett. 90, 014101 (2003).

[13] G. Hu and Z. Qu, Phys. Rev. Lett. 72, 68 (1994).

[14] L. Kocarev, Z. Tasev, and U. Parlitz, Phys. Rev. Lett. 79, 51 (1997).

[15] R. O. Grigoriev, M. C. Cross, and H. G. Schuster, Phys. Rev. Lett. 79, 2795 (1997).

[16] P. Parmananda, Phys. Rev. E 56, 1595 (1997).

[17] S. Boccaletti, J. Bragard, F. T. Arecchi, and H. L. Mancini, Phys. Rev. Lett. 83, 536 (1999).

[18] H. Chaté, A. Pikovsky, and O. Rudzick, Physica D 131, 17 (1999).

[19] L. Junge and U. Parlitz, Phys. Rev. E 62, 438 (2000).

[20] M. G. Rosenblum and A. Pikovsky, Phys. Rev. E 64, 045202 (2001).

[21] M. G. Rosenblum, L. Cimponeriu, A. Bezerianos, A. Patzak, and R. Mrowka, Phys. Rev. E 65, 041909 (2002).

[22] J. Bragard, S. Boccaletti, and H. Mancini, Phys. Rev. Lett. 91, 064103 (2003).

[23] For a comprehensive review on pattern dynamics emerging from space-time bifurcations see M. Cross and P. Hohenberg, Rev. Mod. Phys. 65, 851 (1993), and references therein.

[24] P. Coullet, L. Gil, and F. Roca, Opt. Commun. 73, 403 (1989).

[25] P. Kolodner, S. Slimani, N. Aubry, and R. Lima, Physica D
85, 165 (1995).

[26] Y. Kuramoto and S. Koga, Suppl. Prog. Theor. Phys. 66, 1081 (1981).

[27] T. Leweke and M. Provansal, Phys. Rev. Lett. 72, 3174 (1994).

[28] B. D. Josephson, Phys. Lett. 1, 251 (1962).

[29] I. Aranson and L. Kramer, Rev. Mod. Phys. 74, 99 (2002).

[30] B. I. Shraiman, A. Pumir, W. van Saarlos, P. C. Hohenberg, H. Chaté, and M. Holen, Physica D 57, 241 (1992).

[31] H. Chaté, Nonlinearity 7, 185 (1994).

[32] H. Chaté, in Spatiotemporal Patterns in Nonequilibrium Complex Systems, edited by P. E. Cladis and P. Palffy-Muhoray (Addison-Wesley, New York, 1995).

[33] B. Janiaud, A. Pumir, D. Bensimon, V. Croquette, H. Richter, and L. Kramer, Physica D 55, 269 (1992).

[34] A. Torcini, H. Frauenkron, and P. Grassberger, Phys. Rev. E 55, 5073 (1997).

[35] A. Torcini, Phys. Rev. Lett. 77, 1047 (1996).

[36] M. van Hecke, Physica D 174, 134 (2003).

[37] L. Brusch, A. Torcini, and M. Bär, Physica D 174, 152 (2003).

[38] S. Boccaletti, J. Bragard, and F. T. Arecchi, Phys. Rev. E 59, 6574 (1999).

[39] J. Bragard and S. Boccaletti, Phys. Rev. E 62, 6346 (2000).

[40] J. Bragard, S. Boccaletti, and F. T. Arecchi, Int. J. Bifurcation Chaos Appl. Sci. Eng. 11, 2715 (2001).

[41] S. Ducci, P. L. Ramazza, W. Gonzales-Vinas, and F. T. Arecchi, Phys. Rev. Lett. 83, 5210 (1999).

[42] J. Lega, B. Janiaud, S. Jucquois, and V. Croquette, Phys. Rev. A 45, 5596 (1992).

[43] J. Perraud, A. De Witt, E. Dulos, P. De Kepper, G. Dewel, and P. Borckmans, Phys. Rev. Lett. 71, 1272 (1993).

[44] W. Zurek, Phys. Rep. 276, 177 (1996).

[45] A. Abrikosov, Fundamentals of the Theory of Metals (Elsevier Science, New York, 1988).

[46] W. H. Press et al., Numerical Recipes in Fortran 90 (Cambridge University Press, Cambridge, 1992).

[47] D. A. Egolf and H. S. Greenside, Phys. Rev. Lett. 74, 1751 (1995).

[48] L. Brusch, M. G. Zimmermann, M. van Hecke, M. Bär, and A. Torcini, Phys. Rev. Lett. 85, 86 (2000).

[49] H. Sakaguchi, Prog. Theor. Phys. 83, 169 (1990).

[50] H. Sakaguchi, Prog. Theor. Phys. 84, 792 (1990).

[51] B. Blasius, A. Huppert, and L. Stone, Nature (London) 399, 354 (1999).

[52] O. Bjørnstad and J. Bascompte, J. Anim. Ecol. 70, 924 (2001). 